\title{
The Yangbajing Super Complex Array Plan Based on the ARGO Carpet
}

Youheng Tan and Min Zha ${ }^{\mathrm{a}}$

${ }^{a}$ Key Laboratory of astroparticle and cosmic ray, Institute of High Energy Physics, YuQuan Road 19 B, 100049 Beijing, P.R.China

Following the completion of the $5700 \mathrm{~m}^{2}$ RPC carpet-like AS array at YangBaJing this year, a further plan based on the ARGO Carpet is raised. It characterizes with its high altitude site, its full-coverage detector array and the multi-parameter measurements. Apart from studies on $\mathrm{TeV}$ and sub-TeV energy range, UHE Gamma-ray sources and the Knee Physics will become its characteristic subjects.

In the first phase of the plan, the Yangbajing Super Complex Array (YSCA) will include a $10^{4} \mathrm{~m}^{2}$ RPC Carpet, five indoor $\mu$-detectors $\left(\sim 170 \mathrm{~m}^{2}\right.$ each), four big outdoor $\mu$-detectors $\left(\sim 432 \mathrm{~m}^{2}\right.$ each $)$ and a traditional field scintillation detector array surrounding the Carpet. Monte-Carlo study shows that, with such an array in YBJ (4300m a.s.l.), $\gamma$-ray induced air showers can be separated from proton induced air showers clearly using only the observed electron and $\mu$ data event by event. To assist the classification of UHE air showers induced by different primary nuclei groups without severe interference by Composition/Model Entanglement, a second phase plan having a Central Burst Detector Array (CBDA) and some pulse shape detectors is schemed.

Key Words: AS array (Air Shower array), UHE (Ultra High Energy), RPC (Resistive Plate Chamber), Gamma-ray ( $\gamma$-ray), Knee Physics, Muon detector ( $\mu$-detector), IACT (Image Air Cherenkov Telescope)

\section{Indroduction}

The $5700 \mathrm{~m}^{2}$ ARGO carpet array [1] has been completed and operated in the middle of 2006 at YBJ Cosmic Ray Observatory (4300m a.s.l.) by the Chinese-Italian ARGO Collaboration. Many researches will be carried out with it in the $\mathrm{TeV}$ and Sub-TeV energy region as scheduled [2]. Since the rapid development of IACT [3] and space detector [4] in recent years, ARGO experiment has to advance further to face the new challenge. Under the guidance of GLAST (2007-?), the limitation on FOV and incapacity on unknown objects of IACT will be minimized, the current ARGO array will be difficult to compete with IACTs on $\gamma$-ray astronomy in Sub-TeV and $\mathrm{TeV}$ region, and will be restricted in the new source confirmation, the known source monitoring and the full-sky survey for diffuse $\gamma$-ray. On the other hand, the UHE $(100 \mathrm{TeV}-100 \mathrm{PeV}) \gamma$-ray astronomy and Knee Physics are hard to be essentially approached by space and balloon detectors, and keep as a duty territory of air shower experiments.

The world wide surge of passion on UHE $\gamma$-ray source (therefore cosmic ray source) research in 80 s of last century [5], failed due to their coarse AS array and its inefficiency on selecting primary $\gamma$-rays from the proton background sea, although the shock acceleration in SNRs and diffuse propagation through our galaxy is widely believed to be the dominant mechanism for cosmic ray production up to PeV energy. As to the "knee Physics", it has been the hot point and "hard nuts" over near half century in cosmic ray physics [6]. After the successive efforts with traditional AS array (BASJE [7], Akeno [8]-[9], ANI [10], KASCADE [11]-[12]) and balloon experiments (JECEE [13], RUNJOB[14], ATIC[15], CREAM[16] the kneelike bending is steadily established, but the concrete feature of knee structure and the spectra of different mass groups still keep its inconclusive state and the origin of the knee remains unknown. The poor statistics of balloon experiments and the inefficient identification of AS array experiments on primary composition are responsible for this slow progress. The inefficient identification of AS experiment is due to its indirect measurement feature. It produced two problems. One is that 
individual AS development in the atmosphere suffers big fluctuation. The other one is that the interpretation of most AS data depends on the UHE interaction models. The AS development fluctuation is so severe at low altitude that it can easily dominate over the instrumental resolution of traditional AS arrays; the UHE model uncertainty and the primary particle unknown get us into the Composition/Model entanglement $(\mathrm{C} / \mathrm{M}$ entanglement). How to minimize the shower fluctuation and untie the $\mathrm{C} / \mathrm{M}$ entanglement will become the key route to obtain an accurate UHE cosmic ray all-particle spectrum (as the normalization standard) and several convincing spectra of major cosmic ray element groups.

YBJ Observatory is situated at an altitude near the maximum development of UHE vertical air showers, where the air showers around the Knee have maximum size, minimum fluctuation and less model dependence. These physics advantages are ensured to be brought into full play by its ideal topography, mild climate, full-time traffic (provided by flights, railway and highway), convenient communication (a $155 \mathrm{MB} / \mathrm{s}$ internet link is using for data online transmission), sufficient electric power (supplied by nearby geothermal power plant) as well as the helpful neighboring institutions. Moreover, the new ARGO carpet, is the finest AS array in the world, its unique power on displaying the full picture of the spatial and temporal distribution of the charge particles in individual air shower, may provide us an effective means to separate AS events according its primary composition on the event by event basis (rather than simply a mean mass variation with energy) and open an era of fine measurement of AS experiment to lose the $\mathrm{C} / \mathrm{M}$ entanglement. To strengthen this power, the correlations between different experiment parameters, and therefore a complex array based on the ARGO carpet is necessary.

\section{The frame of the YSCA}

Based on the $\mathrm{e} / \mu$ distributions in $50 \mathrm{TeV}-100$ $\mathrm{PeV}$ region simulated using CORSICA code with QGSJET model, the configuration of YSCA is designed as shown in Fig.1.

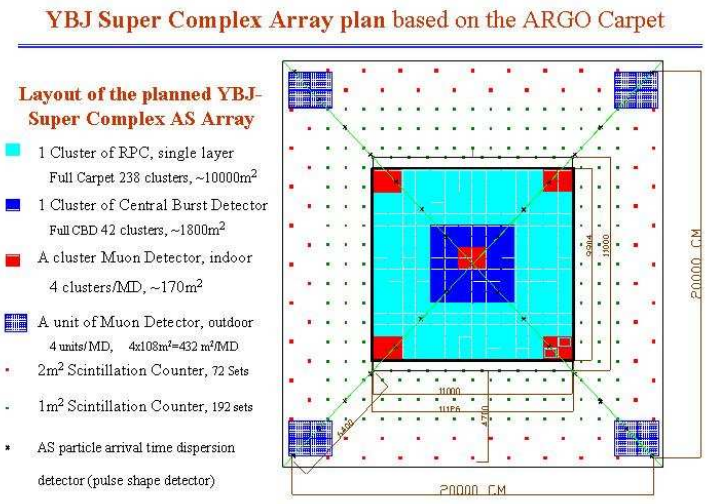

Figure 1. The layout of YBJ Super Complex Array.

1. A Central Carpet made by single layer RPC [17] $\left(\sim 10^{4} \mathrm{~m}^{2}\right.$, in ARGO Hall).

2. An Outer Sampling Array surrounding the carpet, made up of 264 sets of scintillation counters (cover the area of $50 \mathrm{~m}-100 \mathrm{~m}$ from array center).

3. A Muon-Detector Array composed of 5 indoor $\mu$-detectors and 4 outdoor $\mu$-detectors. The indoor $\mu$-detectors will be made with RPC and lead plates (Fig.2), each $170 \mathrm{~m}^{2}$; the outdoor $\mu$-detectors will be made with streamer tubes [18] and lead or iron plates (Fig.3), each $432 \mathrm{~m}^{2}$. The above 3 items compose the basic frame of YSCA.

4. A Central Burst Detector Array (CBDA, $1800 \mathrm{~m}^{2}$ ) made up of RPC and lead plates installed in such a manner without breaking the integrality of the carpet, will be used for model checking and assisting the primary composition determination on some individual air showers.

5. Dozens of pulse shape detectors will be distributed on the diagonals of the array to extract the information of temporal distribution of particles in AS particle disc for 


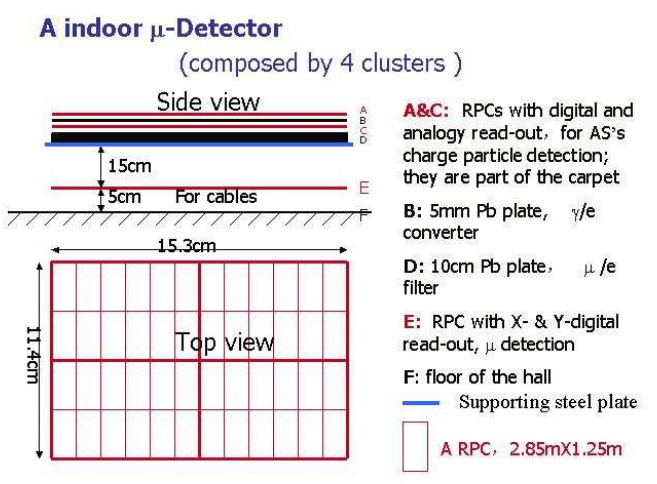

Figure 2. An indoor Muon-detector.

assisting the primary particle composition identification of individual air shower.

\section{Discussion}

One can see from above, all detectors in the array are full-time type and with high event select efficiency (except the CBDA). Their high effective event rate and high accompanying rate are indispensable for correct measurement of UHE cosmic ray spectra.

There are two key points in the preparation of YSCA plan. One is to realize the RPC analogy readout with large dynamic range $\left(\sim 10^{4}\right)$ so that the carpet can be qualified in UHE air shower measurement. In fact, such a front end DAQ system has been equipped to $24 \mathrm{RPC}$ clusters for test running, but the calibration is not yet finished. The second one is to develop and test an effective method to separate the recorded air showers into several primary composition groups, based on the picture pattern of the particle spatial and temporal distribution of individual air shower on the carpet, and accordingly the sensitivity and other performances of array can be calculated properly. Both of them are crucial important for Knee Physics study. As shown by simulation, while the difference of $\mu$ density between a $100 \mathrm{TeV}$ Gamma
One Block of outdoor $\mu$-Detector

(a $\mu$-Detector composed by 4 Blocks )

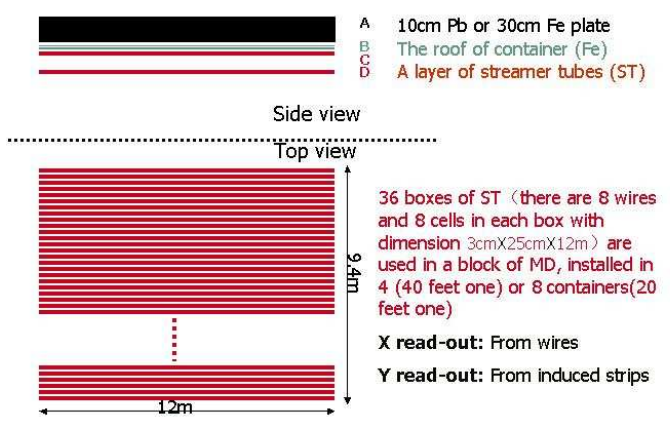

Figure 3. One block of an outdoor Muon detector.

induced AS $(\gamma$-AS) and a proton induced AS (P$\mathrm{AS}$ ) at a core distance $\sim 50 \mathrm{~m}$ is as distinguished as 1:65, it's only 3 times difference between the $\mathrm{P}-\mathrm{AS}$ and iron induced AS (Fe-AS). That means even without the judgment from Carpet, a simple $\mu$-cut can separate the $\gamma$-AS from the P-AS clearly (therefore the UHE $\gamma$-ray source search can be fulfilled fairly using a traditional AS array with big $\mu$-detectors), but it's far from sufficient for Knee Physics. Accordingly, a super complex array at YBJ level with a big carpet is a logical solution. For this prospect, meanwhile, the calibration of RPC analogy readout with an accelerator beam and detailed Monte-Carlo simulations (including the $\mu$-pollution of punch-through effect of AS energetic electrons in near-core region) are underway.

This work is supported in China by NSFC(10120130794), the Chinese Ministry of Science and Technology, the Chinese Academy of Sciences, the Key Laboratory of Particle Astrophysics, CAS, and in Italy by the Istituto Nazionale di Fisica Nucleare (INFN). 


\section{REFERENCES}

1. A.Aloisio et al., Nuovo Cimento 24, N.4-5 (2001) 739

2. Y.H.Tan, Progress in Astronomy 21, No.4 (2003) 318, in Chinese

3. http://mpi-hd.mpg.de/hfm/HESS/HESS.html

4. http://glast.gstc.nasa.gov/

5. Proceeding of the workshop "Techniques in Ultra High Energy Gamma Ray Astronomy", La Jolla, 1985. Edited by R.J.Prothereo and S.A.Stephens

6. G.V.Kulikov and G.B.Khristiansen, Sov.Phys. JETP, 35 (1959) 441

7. F.Kakimoto et al., Proc.17 ${ }^{\text {th }}$ ICRC, Pairs, 11(1981) 254
8. T.Hara et al., Proc. $16^{\text {th }}$ ICRC, Kyoto, 9 (1979) 254

9. Y.H.Tan et al., ICR-Report-99-82-2, (1982)

10. ANI Collaboration, NIM A 323, (1992) 104

11. O.H.Klages et al., Nucl.Phys.B (Proc.suppl.) 52B, (1997) 92

12. T.Antoni et al., Astroparticle Physics 19 (2003) 715

13. http://marge.phy.washington.edu/jacce/

14. http://runjob.boon.ru/

15. http://atic.phys.lsu.rdu/aticweb/

16. http://www.unisi.it/ficica/cream/

17. ARGO Collaboration, Nuclear Instrument and Methods in Physics Research A 508 (2003) 110

18. M.Ambrosio rt al., Astroparticle Physics 4 1995) 33 